Bull. Austral. Math. Soc.

VOL. 67 (2003) [365-375]

\title{
EXTREME POINTS IN SPACES BETWEEN DIRICHLET AND VANISHING MEAN OSCILLATION
}

\author{
K.J. WIRThS AND J. XIAO
}

For $p \in(0, \infty)$ define $Q_{p, 0}(\partial \Delta)$ as the space of all Lebesgue measurable complexvalued functions $f$ on the unit circle $\partial \Delta$ for which $\int_{\partial \Delta} f(z)|d z| /(2 \pi)=0$ and

$$
\frac{1}{(2 \pi)^{2}} \int_{I} \int_{I} \frac{|f(z)-f(w)|^{2}}{|z-w|^{2-p}}|d z||d w|=o\left(|I|^{p}\right)
$$

as the open subarc $I$ of $\partial \Delta$ varies. Note that each $Q_{p, 0}(\partial \Delta)$ lies between the Dirichlet space and Sarason's vanishing mean oscillation space. This paper determines the extreme points of the closed unit ball of $Q_{p, 0}(\partial \Delta)$ equipped with an appropriate norm.

\section{INTRODUCTION}

Denote by $\Delta$ and $\partial \Delta$ the open unit disk and the unit circle in the finite complex plane $\mathbb{C}$, respectively. For $p \in(0, \infty)$, let $Q_{p}(\partial \triangle)$ (respectively $Q_{p, 0}(\partial \Delta)$ ) be the class of all Lebesgue measurable functions $f: \partial \Delta \rightarrow \mathbb{C}$ for which $\int_{\partial \Delta} f(z)|d z| /(2 \pi)=0$ and

$$
S_{p}(f, I):=\frac{1}{(2 \pi)^{2}} \int_{I} \int_{I} \frac{|f(z)-f(w)|^{2}}{|z-w|^{2-p}}|d z||d w|=O\left(|I|^{p}\right) \quad\left(\text { respectively } \quad o\left(|I|^{p}\right)\right)
$$

as $I \subseteq \partial \Delta$ varies. Here and throughout this paper, $I$ means an open subarc of $\partial \triangle$ and $|I|$ stands for the normalised arclength of $I \subseteq \partial \triangle$, that is, $|I|=\int_{I}|d z| /(2 \pi)$. It is clear that $Q_{p, 0}(\partial \Delta) \subseteq Q_{p}(\partial \Delta)$. For convenience, equip $f \in Q_{p}(\partial \triangle)$ with the following norm

$$
\|f\|_{Q_{p}(\partial \Delta)}:=\sup _{I \subseteq \partial \Delta}\left[\frac{S_{p}(f, I)}{|I|^{p}}\right]^{1 / 2} .
$$

So, $Q_{p}(\partial \Delta)$ is a Banach space and $Q_{p, 0}(\partial \Delta)$ is its closed subspace.

In the case $p \in(0,1)$ both classes were introduced in [3] and [6] when Essén, Nicolau, and Xiao studied the boundary behaviour of the holomorphic $Q_{p}$-spaces (see

Received 23rd May, 2002

Research supported by AvH (Germany) and NSERC (Canada). The authors are grateful to S. Axler for interesting discussions.

Copyright Clearance Centre, Inc. Serial-fee code: 0004-9727/03 \$A2.00+0.00. 
[1]). More important is that the article [9] (see [4] for another proof) proved that $Q_{p}(\partial \triangle) \subseteq B M O(\partial \triangle)$ and equality occurs as $p>1$. In Section 4 of our current paper, we shall demonstrate that $Q_{p, 0}(\partial \triangle) \subseteq V M O(\partial \triangle)$ and equality happens again as $p>1$. Here $B M O(\partial \triangle)$ (respectively $V M O(\partial \triangle)$ ) is John-Nirenberg's [5] (respectively Sarason's [8]) space of functions with bounded respectively vanishing mean oscillation on $\partial \triangle$. More precisely, for $q \geqslant 1$ and a Lebesgue measurable function $f: \partial \triangle \rightarrow \mathbb{C}$ we say $f \in B M O(\partial \triangle)$ provided $\int_{\partial \triangle} f(z)|d z| /(2 \pi)=0$ and $\|f\|_{q-M O}=\sup _{z \in \partial \triangle} f_{q}^{\#}(z)<\infty$, where

$$
f_{q}^{\#}(z)=\sup _{z \in I}\left[\frac{1}{2 \pi|I|} \int_{I}\left|f(z)-\frac{1}{2 \pi|I|} \int_{I} f(w)\right| d w||^{q}|d z|\right]^{1 / q},
$$

and here, the supremum is taken over all open subarcs $I \subseteq \partial \triangle$ such that $z \in I$. Moreover we call $f \in V M O(\partial \triangle)$ if $f \in B M O(\partial \triangle)$ and

$$
\lim _{\delta \searrow 0} \sup _{|I|<\delta} \frac{1}{2 \pi|I|} \int_{I}\left|f(z)-\frac{1}{2 \pi|I|} \int_{I} f(w)\right| d w||^{q}|d z|=0,
$$

where the supremum ranges through all open subarcs $I \subseteq \partial \triangle$ with $|I|<\delta$.

Motivated by Axler-Shields' work [2], this paper is devoted to an investigation of the extreme points of the closed ball of $Q_{p, 0}(\partial \Delta)$ (as well as $Q_{p}(\partial \Delta)$ ), but also extends those corresponding results on $V M O(\partial \triangle)$ (as well as $B M O(\partial \triangle)$ ). The main results of this note are presented in Section 2. Of particular interest are some examples of the extreme/nonextreme points provided in Section 3. In the meantime, it is worth mentioning that our functions $g_{n}$ constructed as the extreme points of the closed unit ball of $\left(Q_{p, 0}(\partial \triangle),\|\cdot\|_{Q_{p}(\partial \Delta)}\right)$ are still extreme points of the closed unit ball of $\left(V M O(\partial \triangle),\|\cdot\|_{2-M O}\right)$. Besides this, the method of constructing some nonextreme points of the closed unit ball of $\left(Q_{p, 0}(\partial \Delta),\|\cdot\|_{Q_{p}(\partial \Delta)}\right)$ is valid for the space $\left(V M O(\partial \triangle),\|\cdot\|_{q-M O}\right)$. In other words, there are some nonextreme points in the closed unit ball of $\left(V M O(\partial \triangle),\|\cdot\|_{q-M O}\right)$.

\section{RESUlts}

First of all, let us determine the extreme points of the closed unit ball of $Q_{p, 0}(\partial \triangle)$. For $f \in Q_{p}(\partial \triangle), p \in(0, \infty)$, define the function $E_{p}(f, \cdot)$ on $\partial \triangle$ by

$$
E_{p}(f, z):=\sup _{z \in I}\left[\frac{S_{p}(f, I)}{|I|^{p}}\right]^{1 / 2}
$$

where the supremum ranges over all open subarcs $I \subseteq \partial \triangle$ such that $z \in I$. It is easy to establish the formula:

$$
\|f\|_{Q_{p}(\partial \Delta)}=\sup _{z \in \partial \Delta} E_{p}(f, z)
$$


This is due to an obvious fact that $E_{p}(f, \cdot)$ is lower semi-continuous, that is, $\{z$ $\left.\in \partial \triangle: E_{p}(f, z)>t\right\}$ is an open set for every $t>0$. Letting $C(\partial \triangle)$ be the class of all continuous functions $f: \partial \triangle \rightarrow \mathbb{C}$, we can get further information on $E_{p}(f, \cdot)$. More precisely,

LEMma 2.1. Let $p \in(0, \infty)$ and let $f \in Q_{p, 0}(\partial \triangle)$. Then $E_{p}(f, \cdot) \in C(\partial \triangle)$.

Proof: It suffices to prove that $E_{p}(f, \cdot)$ is upper semi-continuous too, namely that $\left\{z \in \partial \triangle: E_{p}(f, z)<t\right\}$ is an open set for every $t>0$. To do this, fix $t>0$ and let $z \in \partial \triangle$ obey $E_{p}(f, z)<t$. If $E_{p}(f, z) \neq 0$, then by $f \in Q_{p, 0}(\partial \triangle)$, there exists a $\delta>0$ such that

$$
\sup _{I \subseteq \partial \triangle i I \mid<\delta} \frac{S_{p}(f, I)}{|I|^{p}} \leqslant\left[E_{p}(f, z)\right]^{2}
$$

Now let $J \subseteq \partial \triangle$ be an open subarc centred at $z$ whose arclength $|J|=\varepsilon$ is very small compared to $\delta$. And, for $w \in J$ suppose $I \subseteq \partial \triangle$ contains $w$. When $|I|<\delta$, one has $S_{p}(f, I) /|I|^{p}<t^{2}$, thanks to the previous estimation. In the case $|I| \geqslant \delta$ take $K=J \cup I$. Then $K$ is an open subarc containing $z$ and hence

$$
\frac{S_{p}(f, I)}{|I|^{p}} \leqslant\left(\frac{|K|}{|I|}\right)^{p} \frac{S_{p}(f, K)}{|K|^{p}} \leqslant\left(\frac{|K|}{|I|}\right)^{p}\left[E_{p}(f, z)\right]^{2}
$$

Further, putting $E_{p}(f, z)=t-\tau, \tau \in(0, t)$, we get that $S_{p}(f, I) /|I|^{p}<t^{2}$ and thus $E_{p}(f, w)<t$ when $w \in J$ and $|J|=\varepsilon<\delta\left[(t /(t-\tau))^{2 / p}-1\right]$. On the other hand, if $E_{p}(f, z)=0$ then via the analysis on the open subarc $K$, we can select an open subarc $J$ centred at $z$ such that $E_{p}(f, w)=0<t$ as $w \in J$. This completes the proof.

TheOREM 2.2. Let $p \in(0, \infty)$ and $f \in Q_{p, 0}(\partial \triangle)$. Then $f$ is an extreme point of the closed unit ball of $Q_{p, 0}(\partial \triangle)$ if and only if $E_{p}(f, \cdot)$ is identical with 1 .

Proof: Assume that $f$ is an extreme point of the closed unit ball of $Q_{p, 0}(\partial \triangle)$. Since $\|f\|_{Q_{p}(\partial \Delta)} \leqslant 1$, one has that $E_{p}(f, z) \leqslant 1$ for all $z \in \partial \Delta$. If $E_{p}(f, \cdot)$ is not identical with 1 , then by Lemma 2.1 , there is an open subarc $J \subseteq \partial \triangle$ such that $\sup _{z \in J} E_{p}(f, z)<1$. Choose a function $g \in Q_{p, 0}(\partial \triangle)$ such that $\|g\|_{Q_{p}(\partial \Delta)}$ $\leqslant 1-\sup _{z \in J} E_{p}(f, z), g=0$ outside $J$, and $g \not \equiv 0$. When $I \subseteq \partial \triangle$ is such that $I \cap J=\emptyset$, we obviously obtain

$$
\frac{S_{p}(f+g, I)}{|I|^{p}}=\frac{S_{p}(f, I)}{|I|^{p}} \leqslant 1
$$

If $I \subseteq \partial \triangle$ ensures $I \cap J \neq \emptyset$, then

$$
\left[\frac{S_{p}(f+g, I)}{|I|^{p}}\right]^{1 / 2} \leqslant\left[\frac{S_{p}(f, I)}{|I|^{p}}\right]^{1 / 2}+\left[\frac{S_{p}(g, I)}{|I|^{p}}\right]^{1 / 2} \leqslant \sup _{z \in J} E_{p}(f, z)+\|g\|_{Q_{p}(\partial \Delta)} \leqslant 1
$$


Thus $\|f+g\|_{Q_{p}(\partial \Delta)} \leqslant 1$. Similarly, $\|f-g\|_{Q_{p}(\partial \Delta)} \leqslant 1$. As $g \not \equiv 0$, those inequalities show that $f$ is not an extreme point of the closed unit ball of $Q_{p, 0}(\partial \Delta)$, contradicting the assumption.

Conversely, let $E_{p}(f, \cdot) \equiv 1$. If $z_{0} \in \partial \Delta$, then there exists a sequence of open subarcs $I_{n}$ containing $z_{0}$ such that $S_{p}\left(f, I_{n}\right) /\left|I_{n}\right|^{p}$ is convergent to 1 . Without loss of generality, let $I_{n}:=\left(a_{n}, b_{n}\right)$, intervals moving counterclockwise. Then, by passing to a subsequence, we may assume that $a_{n} \rightarrow a$ and $b_{n} \rightarrow b$ in the usual sense. After that, let $I_{z_{0}} \subseteq \partial \Delta$ be the open subarc determined by the interval $(a, b)$, that is, $I_{z_{0}}:=(a, b)$. In this sense, $I_{z_{0}}$ is viewed as the limiting open subarc of $I_{n}$. Accordingly, $S_{p}\left(f, I_{z_{0}}\right) /\left|I_{z_{0}}\right|^{p}=1$. Although $I_{z_{0}}$ does not necessarily contain $z_{0}$, it is easy to see that $z_{0}$ belongs to $\bar{I}_{z_{0}}-$ the closure of $I_{z_{0}}-$ a closed subarc $[a, b]$ of $\partial \triangle$. Observe that since $f \in Q_{p, 0}(\partial \triangle), I_{n}$ cannot get small and hence $I_{z_{0}}$ is not empty.

Now suppose $g \in Q_{p, 0}(\partial \Delta)$ and $\|f+g\|_{Q_{p}(\partial \Delta)} \leqslant 1$ and $\|f-g\|_{Q_{p}(\partial \Delta)} \leqslant 1$. In order to prove that $f$ is an extreme point, we must show that $g \equiv 0$. Fix $z_{0} \in \partial \triangle$ with the open subarc $I_{z_{0}}$ constructed above. Thus,

$$
\left.\frac{f(z)-f(w)}{\left(|z-w| /\left|I_{z_{0}}\right|\right)^{(2-p) / 2}}\right|_{I_{z_{0}} \times I_{z_{0}}}
$$

is an element of the unit sphere of $L^{2}\left(I_{z_{0}} \times I_{z_{0}},\left(2 \pi\left|I_{z_{0}}\right|\right)^{-2}|d z \| d w|\right)$. From $\| f$ $+g \|_{Q_{p}(\partial \Delta)} \leqslant 1$, we also know that

$$
\left.\frac{f(z)-f(w)+g(z)-g(w)}{\left(|z-w| /\left|I_{z_{0}}\right|\right)^{(2-p) / 2}}\right|_{I_{z_{0} \times I_{z_{0}}}}
$$

is a member of the closed unit ball of $L^{2}\left(I_{z_{0}} \times I_{z_{0}},\left(2 \pi\left|I_{z_{0}}\right|\right)^{-2}|d z||d w|\right)$, and similarly when $g$ is replaced by $-g$. Notice that

$$
\begin{aligned}
\left.\frac{f(z)-f(w)}{\left(|z-w| /\left|I_{z_{0}}\right|\right)^{(2-p) / 2}}\right|_{I_{z_{0}} \times I_{z_{0}}}=\left.\frac{[f(z)-f(w)]+[g(z)-g(w)]}{2\left(|z-w| /\left|I_{z_{0}}\right|\right)^{(2-p) / 2}}\right|_{I_{z_{0}} \times I_{z_{0}}} \\
+\left.\frac{[f(z)-f(w)]-[g(z)-g(w)]}{2\left(|z-w| /\left|I_{z_{0}}\right|\right)^{(2-p) / 2}}\right|_{I_{z_{0}} \times I_{z_{0}}}
\end{aligned}
$$

and more importantly, that $L^{2}\left(I_{z_{0}} \times I_{z_{0}},\left(2 \pi\left|I_{z_{0}}\right|\right)^{-2}|d z||d w|\right)$ enjoys the property that every point of the unit sphere is an extreme point of the closed unit ball $([7$, p. 84 , problem 16]). So, the last equation implies that $g(z)-g(w)=0$ on $I_{z_{0}} \times I_{z_{0}}$, that is, $g$ is a constant on $I_{z_{0}}$. 
For each $z_{0} \in \partial \triangle$ let $J_{z_{0}}$ denote the largest open subarc covering $I_{z_{0}}$ such that $g \mid J_{z_{0}}$ is constant. Obviously, as $z_{0}, w_{0} \in \partial \triangle, J_{z_{0}} \cap J_{w_{0}} \neq \emptyset$ induces $J_{z_{0}}=J_{w_{0}}$, and so the collection $\left\{J_{z_{0}}\right\}$ can contain at most countably many different open subarcs, because $|\partial \Delta|=1$. Relabel these disjoint open subarcs $\left\{K_{n}\right\}$. The condition $z_{0} \in \bar{I}_{z_{0}}$ implies $\partial \triangle=U \bar{K}_{n}$ (where $\bar{K}_{n}$ stands for the closure of $K_{n}$ ). Thus $A:=\partial \triangle \backslash \cup K_{n}$ consists of all endpoints of all the subarcs $K_{n}$. In particular, $A$ is a closed, countable set. If $A=\emptyset$, then $\left\{K_{n}\right\}$ contains only one element, namely $\partial \triangle$, and thus $g$ is a constant function on $\partial \triangle$. Note that $\int_{\partial \Delta} g(z)|d z| /(2 \pi)=0$. Accordingly, $g \equiv 0$.

It remains to consider the case: $A \neq \emptyset$. Using the Baire Category Theorem, we see that the non-empty, countable, closed set $A$ must have an isolated point $c \in \partial \triangle$. Since $\partial \triangle=\cup \bar{K}_{n}$, we can conclude that there must be two disjoint open subarcs $K_{n}$ and $K_{m}$ such that $c$ is an endpoint for them. Recall that $g \mid K_{n}$ and $g \mid K_{m}$ are constant. If the two constants do not coincide, then $g$ has a jump discontinuity at $c$, which certainly contradicts the condition $g \in Q_{p, 0}(\partial \triangle)$. If the two constants are the same, then $g$ is constant on the open subarc $K_{n} \cup\{c\} \cup K_{m}$, which violates the maximality of the open subarcs $\left\{J_{z_{0}}\right\}_{z_{0} \in \partial \Delta}$. Thus the case $A \neq \emptyset$ cannot occur, and therefore the proof of the theorem is finished.

Next, we shall deal with the extreme points of the closed unit ball of $Q_{p}(\partial \triangle)$.. This part may be considered as a consequence of the proof of the above theorem, although the forthcoming result looks quite different from that of the extreme points of the closed unit ball of $Q_{p, 0}(\partial \triangle)$.

For $f \in Q_{p}(\partial \triangle)$ and $z \in \partial \triangle$, we shall use $E_{p}(f, z)=1^{+}$to denote that $E_{p}(f, z)$ $=1$ and there exists some open subarc $I_{z}$ containing $z$ that gives $S_{p}\left(f, I_{z}\right) /\left|I_{z}\right|^{p}=1$. This means that the supremum defining $E_{p}(f, z)$ is attained.

CoRollary 2.3. Let $p \in(0, \infty)$ and let $f \in Q_{p}(\partial \triangle)$. If $f$ is an extreme point of the closed unit ball of $Q_{p}(\partial \Delta)$ then there exist no two distinct points $z_{1}$ and $z_{2}$ in $\partial \triangle$ such that $E_{p}\left(f, z_{1}\right)<1$ and $E_{p}\left(f, z_{2}\right)<1$. Conversely, if $E_{p}(f, z)=1^{+}$for all $z \in \partial \triangle$ with one possible exception, then $f$ is an extreme point of the closed unit ball of $Q_{p}(\partial \triangle)$.

Proof: Let $f$ be an extreme point of the closed unit ball of $Q_{p}(\partial \Delta)$. Without loss of generality, we may assume that $\|f\|_{Q_{p}(\partial \Delta)}=1$ in that if $\|f\|_{Q_{p}(\partial \Delta)}<1$ then we may select $g=(1-\varepsilon) f$ and $h=(1+\varepsilon) f$, where $0<\varepsilon<\min \left\{1,\|f\|_{Q_{p}(\partial \Delta)}^{-1}-1\right\}$, and hence it turns out from the equation $f=(g+h) / 2$ that $f$ cannot be an extreme point of the closed unit ball of $Q_{p}(\partial \triangle)$. In order to reach our goal, suppose otherwise that there are two distinct points $z_{k} \in \partial \Delta, k=1,2$ such that $E_{p}\left(f, z_{k}\right)<1, k=1,2$; and use $I_{k}, k=1,2$ to denote the two open subarcs of $\partial \Delta$ which have $\left\{z_{1}, z_{2}\right\}$ as endpoints. Define a function $g \in Q_{p}(\partial \Delta)$ by $\left.g\right|_{I_{1}}=\varepsilon_{1},\left.g\right|_{I_{2}}=-\varepsilon_{2}$, where $\varepsilon_{k}>0$, 
$k=1,2$ are chosen so that $\int_{\partial \Delta} g(z)|d z|=0$ and $\|g\|_{Q_{p}(\partial \Delta)} \leqslant 1-\max _{k=1,2} E_{p}\left(f, z_{k}\right)$. Now, let $I$ be an open subarc of $\partial \triangle$. If both $z_{1}$ and $z_{2}$ are not in $I$, then $g$ is constant on $I$ and thus $S_{p}(f+g, I) /|I|^{p}=S_{p}(f, I) /|I|^{p} \leqslant 1$. If one of $z_{k}$, say, $z_{1}$ lies in $I$, then

$$
\left[\frac{S_{p}(f+g, I)}{|I|^{p}}\right]^{1 / 2} \leqslant\left[\frac{S_{p}(f, I)}{|I|^{p}}\right]^{1 / 2}+\left[\frac{S_{p}(g, I)}{|I|^{p}}\right]^{1 / 2} \leqslant E_{p}\left(f, z_{1}\right)+\|g\|_{Q_{p}(\partial \Delta)} \leqslant 1 .
$$

Accordingly, it follows that $\|f+g\|_{Q_{p}(\partial \Delta)} \leqslant 1$. Similarly, $\|f-g\|_{Q_{p}(\partial \Delta)} \leqslant 1$. Because $f$ may be written as the sum of $(f+g) / 2$ and $(f-g) / 2$, the function $f$ is not an extreme point of the closed unit ball of $Q_{p}(\partial \Delta)$, violating the given condition.

On the other hand, if $w \in \partial \triangle$ is such that $E_{p}(f, z)=1^{+}$for all $z \in \partial \triangle \backslash$ $\{w\}$, then to each $z \in \partial \triangle \backslash\{w\}$, there corresponds an open subarc $I_{z}$ such that $z$ $\in I_{z}$ and $S_{p}\left(f, I_{z}\right) /\left|I_{z}\right|^{p}=1$. Now let $g \in Q_{p}(\partial \triangle)$ satisfy $\|f+g\|_{Q_{p}(\partial \Delta)} \leqslant 1$ and $\|f-g\|_{Q_{p}(\partial \Delta)} \leqslant 1$. To complete the proof, we must show $g \equiv 0$. Applying the same reasoning as in the argument for sufficiency of Theorem 2.2, we can prove that $g \mid I_{z}$ is a constant. Since $g$ is locally constant on the connected set $\partial \triangle \backslash\{w\}, g \equiv 0$ as $\int_{\partial \Delta} g(z)|d z| /(2 \pi)=0$.

\section{EXAMPLES}

In this section we present some examples of either extreme points or nonextreme points of the closed unit ball of $Q_{p, 0}(\partial \Delta)$.

EXAMPle 3.1. (Extreme points.) Let $p \in(0, \infty)$, and for integers $n= \pm 1, \pm 2, \ldots$ let $f_{n}(z)=\lambda z^{n}$ where $z \in \partial \triangle$ and $|\lambda| \equiv 1$. Then $g_{n}=f_{n} /\left\|f_{n}\right\|_{Q_{p}(\partial \Delta)}$ are extreme points of the closed unit ball of $Q_{p, 0}(\partial \triangle)$.

Proof: To make these examples more precise, by Theorem 2.2

$$
E_{p}\left(g_{n}, z\right)=1, \quad \forall z \in \partial \triangle .
$$

In fact, some elementary calculations tell us that

$$
S_{p}\left(f_{n}, I\right)=2^{p+1} \int_{0}^{|I|}(|I|-t) \sin ^{p-2}(\pi t) \sin ^{2}(n \pi t) d t
$$

and so that

$$
E_{p}^{2}\left(f_{n}, z\right)=\sup _{|I| \in(0,1]} \frac{2^{p+1}}{|I|^{p}} \int_{0}^{|I|}(|I|-t) \sin ^{p-2}(\pi t) \sin ^{2}(n \pi t) d t .
$$

Thus, $\left\|f_{n}\right\|_{Q_{p}(\partial \Delta)}=E_{p}\left(f_{n}, z\right)$ for each $z \in \partial \triangle$, and then $E_{p}\left(g_{n}, \cdot\right) \equiv 1$ follows.

In the sequel, we point out that not all points on the closed unit ball of $Q_{p, 0}(\partial \triangle)$ are extreme points. In fact, we consider a function first defined on $[0,2 \pi)$ with mean value zero and then extended periodically. 
ExAmPle 3.2. (Nonextreme points) Let $p \in(0, \infty)$, and for $\delta \in(0,1)$ let

$$
f_{\delta}\left(e^{i \theta}\right)=\left\{\begin{array}{cl}
\frac{\theta}{\delta}, & \theta \in[0, \delta] \\
1, & \theta \in[\delta, 2 \pi-\delta] \\
\frac{2 \pi-\theta}{\delta}, & \theta \in[2 \pi-\delta, 2 \pi) .
\end{array}\right.
$$

Also put $g_{\delta}=f_{\delta}-1+\delta /(2 \pi)$ on $[0,2 \pi)$ and extend it $2 \pi$-periodically. Then there exists a $\delta>0$ such that $g_{\delta} /\left\|g_{\delta}\right\|_{Q_{p}(\partial \Delta)}$ is a nonextreme point of the closed unit ball of $Q_{p, 0}(\partial \triangle)$.

PROOF: A key observation is that $g_{\delta}$ is convergent to the zero-function as $\delta \rightarrow 0$. Because $f_{\delta}$ is a Lip1-function, $g_{\delta} /\left\|g_{\delta}\right\|_{Q_{p, 0}(\partial \Delta)}$ is in $Q_{p, 0}(\partial \Delta)$. However, we show that it is not an extreme point of the closed unit ball of $Q_{p, 0}(\partial \triangle)$. By Theorem 2.2 we know this will be done if one can prove that $E_{p}\left(f_{\delta}, \cdot\right)$ is not a constant function for some $\delta$. For this it suffices to verify $E_{p}\left(f_{\delta}, 0\right) \neq E_{p}\left(f_{\delta}, \pi\right)$ for some $\delta$. First, for any open subarc (or subinterval) $I=(a, b) \subseteq(0, \delta)$ we have

$$
\frac{S_{p}\left(f_{\delta}, I\right)}{|I|^{p}}=\frac{2^{p}(2 \pi)^{p-2}}{(b-a)^{p} \delta^{2}} \int_{0}^{b-a} \frac{(b-a-t) t^{2}}{\sin ^{2-p} t / 2} d t .
$$

Thus

$$
\sup _{I \subseteq(0, \delta)} \frac{S_{p}\left(f_{\delta}, I\right)}{|I|^{p}} \geqslant \frac{2^{p}(2 \pi)^{p-2}}{\delta^{2+p}} \int_{0}^{\delta} \frac{(\delta-t) t^{2}}{\sin ^{2-p} t / 2} d t
$$

Furthermore, by Lemma 2.1 and the limit

$$
\lim _{\delta \rightarrow 0} \frac{1}{\delta^{p+2}} \int_{0}^{\delta} \frac{(\delta-t) t^{2}}{\sin ^{2-p} t / 2} d t=\frac{2^{2-p}}{(p+1)(p+2)}
$$

we can find a $\delta_{1} \in(0,1)$ such that for $\delta \in\left(0, \delta_{1}\right)$,

$$
E_{p}\left(f_{\delta}, 0\right)>2^{-1}\left[\frac{2^{p} \pi^{p-2}}{(p+1)(p+2)}\right]^{1 / 2}:=\frac{\mu_{0}}{2}
$$

Second, suppose $I \subseteq \partial \triangle$ is any open subarc containing $\pi$. If $|I| \leqslant(\pi-\delta) /(2 \pi)$, then $S_{p}\left(f_{\delta}, I\right)=0$. If $1 \geqslant|I|>(\pi-\delta) /(2 \pi)$, then by the definition of $f_{\delta}$,

$$
S_{p}\left(f_{\delta}, I\right) \leqslant S_{p}\left(f_{\delta}, \partial \Delta\right)=\frac{1}{(2 \pi)^{2}} \iint_{\Omega} \frac{\left|f_{\delta}\left(e^{i \phi}\right)-f_{\delta}\left(e^{i \psi}\right)\right|^{2}}{\left|e^{i \phi}-e^{i \psi}\right|^{2-p}} d \phi d \psi
$$


where $\Omega$ is a domain defined by $\bigcup_{j=1}^{4} \Omega_{j}$ :

$$
\Omega_{j}= \begin{cases}\{(\phi, \psi): 0 \leqslant \phi \leqslant \delta, 0 \leqslant \psi \leqslant 2 \pi\}, & j=1 \\ \{(\phi, \psi): 2 \pi-\delta \leqslant \phi \leqslant 2 \pi, 0 \leqslant \psi \leqslant 2 \pi\}, & j=2 \\ \{(\phi, \psi): 0 \leqslant \phi \leqslant 2 \pi, 0 \leqslant \psi \leqslant \delta\} & j=3 \\ \{(\phi, \psi): 0 \leqslant \phi \leqslant 2 \pi, 2 \pi-\delta \leqslant \psi \leqslant 2 \pi\}, & j=4 .\end{cases}
$$

It is a completely elementary estimation to obtain a $\delta_{2} \in(0,1)$ such that for $\delta \in\left(0, \delta_{2}\right)$,

$$
\iint_{\Omega_{j}} \frac{\left|f_{\delta}\left(e^{i \phi}\right)-f_{\delta}\left(e^{i \psi}\right)\right|^{2}}{\left|e^{i \phi}-e^{i \psi}\right|^{2-p}} d \phi d \psi \leqslant\left(\frac{\pi-\delta}{2 \pi}\right)^{p}\left(\frac{2 \pi \mu_{0}}{2}\right)^{2}, \quad j=1,2,3,4 .
$$

Hence

$$
\frac{S_{p}\left(f_{\delta}, I\right)}{|I|^{p}} \leqslant \frac{1}{(2 \pi)^{2}}\left(\frac{2 \pi}{\pi-\delta}\right)^{p} \iint_{\Omega} \frac{\left|f_{\delta}\left(e^{i \phi}\right)-f_{\delta}\left(e^{i \psi}\right)\right|^{2}}{\left|e^{i \phi}-e^{i \psi}\right|^{2-p}} d \phi d \psi \leqslant\left(\frac{\mu_{0}}{2}\right)^{2}
$$

Consequently, $E_{p}\left(f_{\delta}, \pi\right) \leqslant \mu_{0} / 2$ whenever $\delta \in\left(0, \delta_{2}\right)$. Therefore there exists a $\delta_{3}$ $\in\left(0, \min \left\{\delta_{1}, \delta_{2}\right\}\right)$ such that $E_{p}\left(f_{\delta_{3}}, 0\right)>\mu_{0} / 2$ and $E_{p}\left(f_{\delta_{3}}, \pi\right) \leqslant \mu_{0} / 2$. This concludes the proof.

Recall that the (boundary) Dirichlet space $D(\partial \triangle)$ consists of all Lebesgue measurable complex-valued functions $f$ on $\partial \triangle$ for which $\int_{\partial \Delta} f(z)|d z| /(2 \pi)=0$ and

$$
\|f\|_{D(\partial \Delta)}:=\left[\frac{1}{(2 \pi)^{2}} \int_{\partial \Delta} \int_{\partial \Delta} \frac{|f(z)-f(w)|^{2}}{|z-w|^{2}}|d z \| d w|\right]^{1 / 2}<\infty .
$$

It is clear that $D(\partial \triangle) \subseteq \bigcap_{p>0} Q_{p, 0}(\partial \Delta)$, and that every point on the closed unit ball of $\left(D(\partial \Delta),\|\cdot\|_{D(\partial \Delta)}\right)$ is an extreme point and vice versa. An explicit computation involving $E_{p}\left(f_{\delta}, \cdot\right)$ above reveals that for $p$ small one has to choose $\delta$ small in order to get a nonextreme point of the closed unit ball of $\left(Q_{p, 0}(\partial \Delta),\|\cdot\|_{Q_{p}(\partial \Delta)}\right)$. This reflects the fact that $Q_{p, 0}(\partial \triangle)$ approaches $D(\partial \triangle)$ as $p \searrow 0$ in some sense.

\section{APPENDIX}

The first result of this section is to illustrate the important basic relationship between $Q_{p, 0}(\partial \triangle)$ and $V M O(\partial \triangle)$ mentioned in the introduction.

PRoposition 4.1. If $0<p_{1}<p_{2}<\infty$ then $Q_{p_{1}, 0}(\partial \triangle) \subseteq Q_{p_{2}, 0}(\partial \triangle)$. In particular, if $p \in(1, \infty)$ then $Q_{p, 0}(\partial \triangle)=V M O(\partial \triangle)$.

Proof: It suffices to show that each $Q_{p, 0}(\partial \Delta)$ coincides with $V M O(\partial \triangle)$ whenever $p>1$. First, we verify $V M O(\partial \Delta) \subseteq Q_{p, 0}(\partial \triangle)$. To do so, we observe the 
integrated Lip-character of $Q_{p, 0}(\partial \triangle)$ (which may be worked out via the change of variables; see also [4, p. 579]): $f \in Q_{p, 0}(\partial \triangle)$ if and only if $\lim _{\delta \rightarrow 0} F_{p}(f, \delta)=0$, where for $\delta \in(0,1)$,

$$
F_{p}(f, \delta):=\sup _{I \subseteq \partial \Delta,|I|<\delta}|I|^{-p} \int_{0}^{|I|} \sin ^{p-2} \frac{\pi t}{2} \int_{I}\left|f\left(e^{i(s+t)}\right)-f\left(e^{i s}\right)\right|^{2} d s d t .
$$

For convenience, we use $U \lesssim V$ to denote that there is a constant $c>0$ such that $U \leqslant c V$. If $U \lesssim V$ and $V \lesssim U$ hold simultaneously then we say that $U \sim V$. In addition, we write $r I(r>0)$ for the open arc with length $r|I|$ and the same centre as $I$, and $f_{J}$ the average of $f$ over $J \subseteq \partial \triangle: f_{J}=(2 \pi|J|)^{-1} \int_{J} f\left(e^{i t}\right) d t$. Now if $f$ in $V M O(\partial \triangle)$ then for any small $\varepsilon>0$ there is a $\delta \in(0,1 / 3)$ such that as $|I|<\delta$,

$$
\int_{3 I}\left|f\left(e^{i s}\right)-f_{3 I}\right|^{2} d s<2 \pi \varepsilon|I|
$$

and hence

$$
\int_{0}^{|I|} \sin ^{p-2} \frac{\pi t}{2} d t \int_{I}\left|f\left(e^{i s}\right)-f_{3 I}\right|^{2} d s \lesssim|I|^{p-1} \int_{3 I}\left|f\left(e^{i s}\right)-f_{3 I}\right|^{2} d s \lesssim \varepsilon|I|^{p} .
$$

Consequently

$$
\int_{0}^{|I|} \sin ^{p-2} \frac{\pi t}{2} \int_{I}\left|f\left(e^{i(t+s)}\right)-f_{3 I}\right|^{2} d s d t \lesssim \varepsilon|I|^{p} .
$$

So, $\lim _{\delta \rightarrow 0} F_{p}(f, \delta)=0$, namely, $f \in Q_{p, 0}(\partial \triangle)$.

Second, we show that $Q_{p, 0}(\partial \triangle) \subseteq V M O(\partial \triangle)$. In case $p \in(1,2]$, the result follows immediately from the definition. It remains to consider the case $p>2$. Let $f \in Q_{p, 0}(\partial \triangle)$. Then for arbitrarily small $\varepsilon>0$ there exists a $\delta \in(1,1 / 2)$ such that $S_{p}(f, J)<\varepsilon|J|^{p}$ whenever $|J|<\delta$. Thus for $I \subseteq \partial \triangle$ with $|I|<\delta$, one has

$$
\begin{aligned}
\int_{I} \int_{I}\left|f\left(e^{i s}\right)-f\left(e^{i t}\right)\right|^{2} d s d t & \leqslant \sum_{k=1}^{\infty} \iint_{2^{-k}<\frac{|s-t|}{|I|} \leqslant 2^{1-k}} \frac{\left|e^{i s}-e^{i t}\right|^{2-p}\left|f\left(e^{i s}\right)-f\left(e^{i t}\right)\right|^{2}}{\left|e^{i s}-e^{i t}\right|^{2-p}} d s d t \\
& \lesssim \sum_{k=1}^{\infty}\left(\frac{|I|}{2^{k}}\right)^{2-p} \iint_{|s-t| /|I| \leqslant 2^{1-k}} \frac{\left|f\left(e^{i s}\right)-f\left(e^{i t}\right)\right|^{2}}{\left|e^{i s}-e^{i t}\right|^{2-p}} d s d t \\
& \lesssim \sum_{k=1}^{\infty}\left(\frac{|I|}{2^{k}}\right)^{2-p} \int_{2^{2-k} I} \int_{2^{2-k_{I}}} \frac{\left|f\left(e^{i s}\right)-f\left(e^{i t}\right)\right|^{2}}{\left|e^{i s}-e^{i t}\right|^{2-p}} d s d t \\
& \lesssim \varepsilon|I|^{2},
\end{aligned}
$$

which implies that $f$ in $V M O(\partial \triangle)$. Therefore, the proof is complete.

The second conclusion of this section is an estimate of the distance from $f$ $\in Q_{p}(\partial \triangle)$ to $Q_{p, 0}(\partial \triangle)$. 
PROPOSITION 4.2. Let $p \in(0, \infty)$ and $f \in Q_{p}(\partial \triangle)$ with

$$
d\left(f, Q_{p, 0}(\partial \triangle)\right):=\inf \left\{\|f-g\|_{Q_{p}(\partial \Delta)}: g \in Q_{p, 0}(\partial \triangle)\right\} .
$$

Then

$$
d\left(f, Q_{p, 0}(\partial \triangle)\right) \sim M_{p}(f):=\lim _{\delta \rightarrow 0} \sup _{I \subseteq \partial \Delta,|I|<\delta}\left[\frac{S_{p}(f, I)}{|I|^{p}}\right]^{1 / 2} .
$$

Proof: Since $d\left(f, Q_{p, 0}(\partial \triangle)\right)=0$ whenever $f \in Q_{p, 0}(\partial \triangle)$, it is easy to show that

$$
M_{p}(f) \leqslant d\left(f, Q_{p, 0}(\partial \triangle)\right), \quad f \in Q_{p}(\partial \triangle) .
$$

Regarding the reversed estimate, we define the function

$$
f_{r}\left(e^{i s}\right)=\frac{1}{2 \pi} \int_{\partial \Delta} f(\eta) \frac{1-r^{2}}{\left|\eta-r e^{i s}\right|^{2}}|d \eta|
$$

for $r \in(0,1)$ and $f \in Q_{p}(\partial \triangle)$. It is clear that $f_{r} \in Q_{p, 0}(\partial \triangle)$ and

$$
f(\zeta)-f_{r}(\zeta)=\frac{1}{2 \pi} \int_{\partial \Delta}[f(\zeta)-f(\zeta \bar{\lambda})] \frac{1-r^{2}}{|1-r \bar{\lambda}|^{2}}|d \lambda|, \quad \zeta=e^{i s}, \quad \lambda=\zeta \bar{\eta}
$$

Setting $T_{\lambda} f(\zeta)=f(\zeta \bar{\lambda})$ and using Minkowski's inequality, we see that for any small $\varepsilon>0$,

$$
\begin{aligned}
\left\|f-f_{r}\right\|_{Q_{p}(\partial \Delta)} & \\
& \lesssim \int_{\partial \Delta}\left\|f-T_{\lambda} f\right\|_{Q_{p}(\partial \Delta)} \frac{1-r^{2}}{|1-r \bar{\lambda}|^{2}}|d \lambda| \\
& \lesssim \int_{|\lambda|<\varepsilon}\left\|f-T_{\lambda} f\right\|_{Q_{p}(\partial \Delta)} \frac{1-r^{2}}{|1-r \bar{\lambda}|^{2}}|d \lambda|+\|f\|_{Q_{p}(\partial \Delta)} \int_{\varepsilon \leqslant|\lambda| \leqslant \pi} \frac{1-r^{2}}{|1-r \bar{\lambda}|^{2}}|d \lambda| \\
& :=\operatorname{Term}_{1}+\operatorname{Term}_{2} .
\end{aligned}
$$

Suppose $\delta \in(0,1)$. By the Lebesgue Dominated Convergence Theorem we know that

$$
\lim _{\lambda \rightarrow 0} \sup _{|I| \geqslant \delta} \frac{S_{p}\left(f-T_{\lambda} f, I\right)}{|I|^{p}}=0 .
$$

Also the Triangle Inequality yields

$$
\sup _{|I|<\delta} \frac{S_{p}\left(f-T_{\lambda} f, I\right)}{|I|^{p}} \lesssim \sup _{|I|<\delta} \frac{S_{p}(f, I)}{|I|^{p}} .
$$


Therefore, if $\varepsilon \rightarrow 0$ then

$$
\operatorname{Term}_{1} \lesssim \sup _{|I|<\delta}\left[\frac{S_{p}(f, I)}{|I|^{p}}\right]^{1 / 2}
$$

And, if $r \rightarrow 1$ then Term $_{2} \rightarrow 0$ and thus

$$
d\left(f, Q_{p, 0}(\partial \triangle)\right) \leqslant \lim _{r \rightarrow 1}\left\|f-f_{r}\right\|_{Q_{p}(\partial \Delta)} \lesssim M_{p}(f)
$$

We are done.

With the help of Proposition 4.1, we see that Proposition 4.2 extends Sarason's vanishing mean oscillation-version in [8]. Of course, Proposition 4.2 derives that $f$ $\in Q_{p, 0}(\partial \triangle)$ if and only if $d\left(f, Q_{p, 0}(\partial \triangle)\right)=0$.

\section{REFERENCES}

[1] R. Aulaskari, J. Xiao and R. Zhao, 'On subspaces and subsets of BMOA and UBC', Analysis 15 (1995), 101-121.

[2] S. Axler and A. Shields, 'Extreme points in VMO and BMO', Indiana Univ. Math. J. 31 (1982), 1-6.

[3] M. Essén and J. Xiao, 'Some results on $Q_{p}$ spaces, $0<p<1$ ', J. Reine Angew. Math. 485 (1997), 173-195.

[4] M. Essén, S. Janson, L. Peng and J. Xiao, 'Q spaces of several real variables', Indiana Univ. Math. J. 49 (2000), 576-615.

[5] F. John and L. Nirenberg, 'On functions of bounded mean oscillation', Comm. Pure Appl. Math. 14 (1961), 415-426.

[6] A. Nicolau and J. Xiao, 'Bounded functions in Möbius invariant Dirichlet spaces', $J$. Funct. Anal. 150 (1997), 383-425.

[7] W. Rudin, Functional analysis (McGraw-Hill, N.J., 1973).

[8] D. Sarason, 'Functions of vanishing mean oscillation', Trans. Amer. Math. Soc. 207 (1975), 391-405.

[9] J. Xiao, 'Some essential properties of $Q_{p}(\partial \triangle)$-spaces', J. Fourier Anal. Appl. 6 (2000), 311-323.

Institute of Analysis

TU-Braunschweig

D-38106 Braunschweig

Germany

e-mail: kjwirths@tu-bs.de
Department of Mathematics and Statistics Memorial University of Newfoundland St. John's, NL A1C 5S7

Canada

e-mail: jxiao@math.mun.ca 